\title{
Neonatal meningitis caused by Citrobacter koseri
}

\author{
R. J. GROSS, B. ROWE, AND J. A. EASTON \\ From the Salmonella and Shigella Reference Laboratory, Central Public Health Laboratory, Colindale, London, \\ and Wexham Park Hospital, Slough, Bucks
}

SYNOPSIS Three cases of neonatal meningitis, two of which were fatal, occurred in a premature baby unit during a period of one week. A fourth case occurred in the same unit six months later. Citrobacter koseri was isolated from the cerebrospinal fluid of all four cases. Detailed biochemical and serological examination of the organisms showed that two distinct bioserotypes were involved.

The final taxonomic position of the organisms variously described as Citrobacter koseri (Frederiksen, 1970), Citrobacter diversus (Ewing and Davis, 1972), and Levinea malonatica (Young, Kenton, Hobbs, and Moody, 1971) has yet to be determined. Their clinical importance is also uncertain, although it seems clear that such organisms occur frequently in clinical specimens. Ewing and Davis (1972) studied 137 strains from human material, but details were not given concerning the site of infection. Frederiksen (1970) described 30 strains, one of which was from cerebrospinal fluid, 10 were from blood cultures, and five were from infected wounds. Young et al (1971) examined 58 isolates from human nose, urine, sputum, and faecal specimens, but none was from blood or cerebrospinal fluid. Booth and McDonald (1971) studied 40 strains, mostly from urine, sputum, and pressure sores. They suggested that the organisms act mainly as secondary invaders.

The study of four cases of neonatal meningitis in a single premature baby unit led to the isolation of two serologically distinct strains of Citrobacter koseri.

\section{Case Reports}

Three babies in the same premature baby unit developed meningitis during their first week of life, and all three were born within a one-week period. Two of the babies had forceps deliveries and the third was delivered by Caesarian section necessitated by placenta praevia. The mothers of two of the babies were in the same antenatal ward.

Each baby presented with the clinical signs of meningitis on its fifth day after birth. Lumbar punctures were performed and a pure culture of a Received for publication 1 December 1972.
Gram-negative organism was grown from each cerebrospinal fluid specimen. Each baby was given intrathecal colistin (up to 6000 units daily), together with intramuscular chloramphenicol and colistin. All three patients also received ampicillin and cloxacillin.

The condition of one baby deteriorated rapidly, and death occurred within 36 hours of the onset of meningitis. In this case an organism identical to that from the cerebrospinal fluid was grown from blood culture. The second baby survived for severaf days, and the original organism was not grown from repeat cerebrospinal fluid specimens, but two different organisms (Escherichia coli and Klebsiella) were isolated. This baby died three weeks after the initial onset of meningitis. The third baby survived and a repeat examination of the cerebrospinal fluid was made after 10 days' treatment. The only abnormality was a raised protein level $(80 \mathrm{mg} / 100 \mathrm{ml})$, and the culture was sterile. Because of the relationship between the three cases, the premature baby unit and the maternity ward were closed and cleaned. No further cases were discovered until six months later.

The fourth baby was born after labour was induced by rupture of the membranes for preeclamptic toxaemia at 37 weeks. Meningitis developed on the seventh day after birth, and lumbar puncture was performed. A Gram-negative organism was isolated, and the baby was treated with intramuscular kanamycin and ampicillin and with intrathecal colistin. The response to treatment was slow and subsequently further courses of chloramphenicol, colistin, gentamicin, and cephaloridine were given

\section{Bacteriological Examination}

The organisms isolated from the cerebrospinal fluid 
of all four cases, and from the blood culture of one case, were motile, Gram-negative bacilli. They were oxidase negative, catalase positive, fermentative, and nitrate reducing. Glucose was fermented with production of acid and gas. Lactose was fermented after three or four days. Mannitol, salicin, dulcitol, adonitol, sorbitol, arabinose, rhamnose, xylose, trehalose, glycerol, cellobiose, sorbose, and maltose were fermented. Inositol, raffinose, and inulin were not fermented. The methyl red test was positive and the Voges-Proskauer test was negative. Indole was produced from peptone water cultures. No growth occurred in potassium cyanide. Simmons citrate was utilized. Tests for arginine and ornithine decarboxylase were positive, while that for lysine decarboxylase was negative. The malonate test was positive, and the phenylpyruvic acid test was negative. $\mathrm{H}_{2} \mathrm{~S}$ was not produced in triple sugar iron agar, and the gluconate test was negative. Urease was not detected in Christensen's medium, and gelatin was not liquefied after 28 days.

It was considered that the organisms were all of the same species and that the biochemical reactions were consistent with the definitions of Citrobacter koseri, Citrobacter diversus, and Levinea malonatica, all of which are synonymous. All isolates were not identical, however. Those from three patients fermented sucrose in 24 hours, while those from one patient (one of the original three cases) failed to ferment sucrose after 14 days.

Antisera were prepared in rabbits using heattreated suspensions of one sucrose-fermenting, and one non-sucrose-fermenting isolate. Heat-treated suspensions of all isolates were then tested by titration against both antisera. All sucrose-fermenting isolates were agglutinated to titre by the antiserum prepared using a sucrose-fermenting strain, but no agglutination was obtained in the antiserum prepared by using a non-sucrose-fermenting strain. Conversely, the antiserum prepared using a nonsucrose-fermenting strain agglutinated only nonsucrose-fermenting isolates. It was concluded that two distinct bioserotypes of a single species were involved in the cases of meningitis studied.

Antibiotic sensitivity tests were carried out on a representative isolate from each of the four cases. The tests were performed on Diagnostic Sensitivity Test Agar (Oxoid CM 261) with Mast ring discs containing the following antibiotics: penicillin (1 unit), ampicillin (25 $\mu \mathrm{g})$, cloxacillin $(5 \mu \mathrm{g})$, sulphonamide $(200 \mu \mathrm{g})$, colistin $(50 \mu \mathrm{g})$, chloramphenicol $(25 \mu \mathrm{g})$, kanamycin $(30 \mu \mathrm{g})$, gentamicin $(10 \mu \mathrm{g})$, cephalexin $(30 \mu \mathrm{g})$, cephaloridine $(25 \mu \mathrm{g})$, streptomycin $(10 \mu \mathrm{g})$, and tetracycline $(25 \mu \mathrm{g})$. In each case the organism was resistant to penicillin, ampicillin, cloxacillin, and sulphonamide, but sensitive to colistin, chloramphenicol, kanamycin, gentamicin, cephalexin, cephaloridine, streptomycin, and tetracycline.

\section{Discussion}

Our report shows that Citrobacter koseri can cause meningitis. The portal of entry in these cases is uncertain, but it is of interest that three of the four cases were forceps deliveries, and the fourth was delivered by Caesarian section. The epidemiology of the cases described is not clear; what appeared at first sight to be a small outbreak of meningitis was shown to involve two serologically distinct organisms. Furthermore, numerous swabs from the mothers and the areas around their beds, from the staff, and from the creams, solutions, and soaps used throughout the maternity unit failed to grow the organism.

Laboratories may encounter these organisms in clinical specimens and should be aware of their possible significance. Their biochemical reactions are similar to those of other members of the Enterobacteriaceae, particularly to Citrobacter freundii, Enterobacter spp, and Escherichia coli. Bacteriologists should be alert to the possibility of incorrect classification.

We would like to thank the paediatricians of the Windsor group of hospitals and Dr E. Sayle, senior pathologist at King Edward VII Hospital, Windsor, for their kind cooperation.

\section{References}

Booth, E. V., and McDonald, S. (1971). A new group of Enterobacteria, possibly a new Citrobacter sp. J. med. Microbiol., 4, 329-336.

Ewing, W. H., and Davis, B. R. (1972). Biochemical characterisation of Citrobacter diversus (Burkey) Werkman and Gillen and designation of the neotype strain. Int. J. syst. Bact., 22, 12-18.

Frederiksen, W. (1970). Citrobacter koseri (n. sp.) a new species within the genus Citrobacter, with a comment on the taxonomic position of Citrobacter intermedium (Werkman and Gillen). Spisy prir. Fak. Univ. Brne, 47, 89-94.

Young, V. M., Kenton, D. M., Hobbs, B. J., and Moody, M. R. (1971). Levinea, a new genus of the family Enterobacteriaceae. Int. J. s.yst. Bact., 21, 58-63. 